\title{
The landscape of genotype-phenotype correlation in AML
}

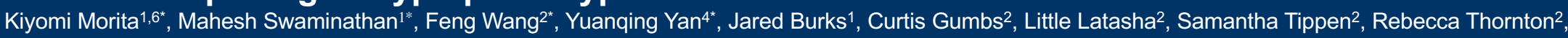

Marcus Coyle ${ }^{2}$, Jianhua Zhang ${ }^{2,5}$, Song Xingzhi ${ }^{2}$, Courtney DiNardo', Elias Jabbour ${ }^{1}$, Michael Andreeff', Jorge Cortes ${ }^{1}$, Marina Konopleva' ${ }^{1}$, Keyur Patel ${ }^{3}$,

Guillermo Garcia-Manero ${ }^{1}$, Hagop Kantarjian ${ }^{1}$, Carlos Bueso-Ramos ${ }^{3}$, Andrew P Futreal ${ }^{2}$, and Koichi Takahashi ${ }^{1} 2^{\star}$

1Department of

${ }^{4}$ Department of Bioinformatics, The University of Texas Health Science Center at Houston, $T X,{ }^{6}$ Department of Hematology and Oncology, The University of Tokyo Graduate School of Medicine

MDAnderson GancerCenter

Making Cancer History

\section{Background}

Acute myeloid leukemia (AML) is a group of clinically

heterogeneous diseases.

Heterogeneous presentation of $A M L$ is defined by the equally

heterogeneous genetic basis during leukemogenesis.

\section{Objective}

To describe the distinct mutation landscape based on the ontogeny of AML.

To describe the correlation between clinical phenotype and genotype in AML.

\section{Methods}

- Bone marrow samples from $536 \mathrm{AML}$ patients were analyzed by targeted capture exome sequencing of 295 genes $(\mathrm{N}=419)$ or

whole exome sequencing $(\mathrm{N}=117)$.

Extensive clinical-genotype correlation was performed using well annotated clinical data.

\section{Results}

Table1. Patient Characteristics

\begin{tabular}{|l|l|l|}
\hline Characteristics & Median & IQR \\
\hline
\end{tabular} \begin{tabular}{|c|c|c|}
\hline WBC & 5.4 & $2.2-21.9$ \\
\hline
\end{tabular}

HGB

PLT

BM blast \%

PB blast

Forritin

Ferritin

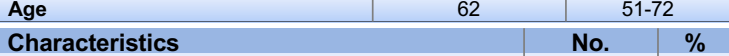

Diagnosis

\begin{tabular}{|l|l|l|}
\hline Diagnosis & & \\
\hline de-novo / secondary/Tx-related AML & $408 / 128$ & $76 / 24$ \\
\hline
\end{tabular}

Prior therapy

\begin{tabular}{|l|l|l|}
\hline untreated/relapse/refractory & $411 / 125$ & $77 / 23$ \\
\hline
\end{tabular}

Cytogenetic risk, ELN defined

\begin{tabular}{|l|l|l|l|}
\hline favorable/intermediate/adverse & $10 / 326 / 177$ & $2 / 61 / 33$ \\
\hline
\end{tabular}

Induction chemotherapy (previously untreated patients only)

\begin{tabular}{|l|c|c|}
\hline High intensity chemotherapy (Ida + AraC-based) & 204 & 50 \\
\hline Low intensity chemotherapy (low dose AraC-based) & 86 & 21 \\
\hline Hypomethylating agents & 91 & 22 \\
\hline
\end{tabular}

Figure 1. Landscape of driver mutations

Clonality estimated based on cancer cell fraction (CCF)

clonal $(\mathrm{CCF} \geq 0.85)$

subclonal minimally (CCF 0.2 and $\mathrm{CCF}<0.85$ )

subclonal $(\mathrm{CCF}<0.2)$
Figure 2. Distinct patterns of somatic mutations based on the ontogeny of AML
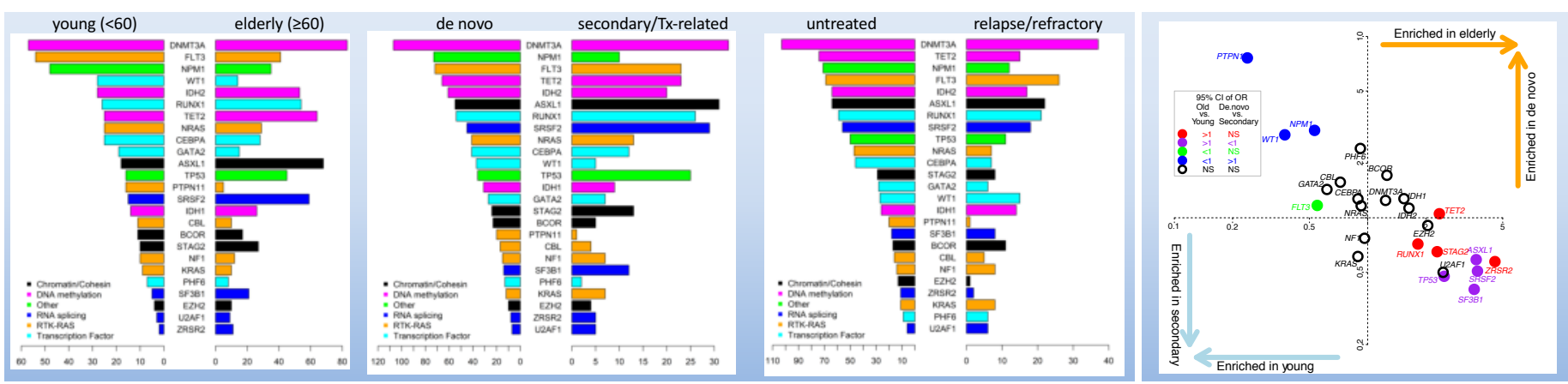

Figure 3. Correlation between somatic mutations and clinical phenotype (BM morphology, PB count, karyotype, immunophenotype based on flow cytometry)
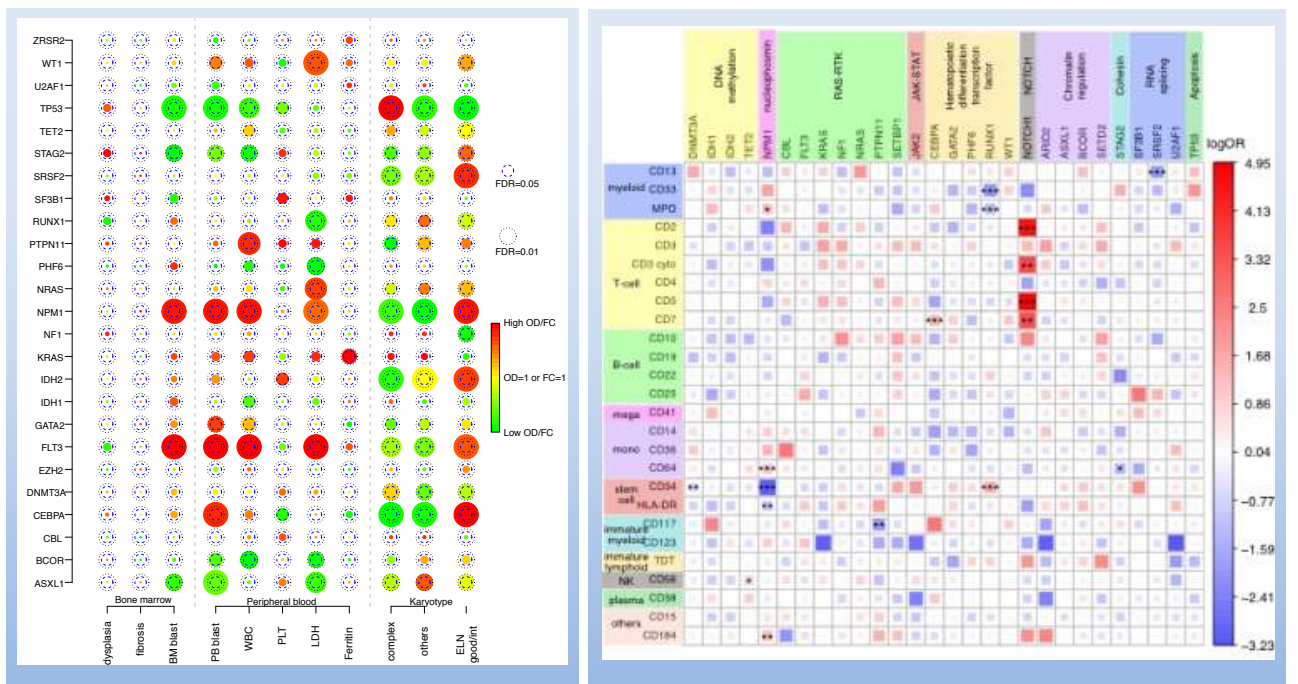

Figure 4. Increased MYC expression in patients with $M Y C$ mutations

- We detected rare mutations in MYC (8 hotspot in $9(2 \%)$ patients.

Patients with MYC mutation showed significantly higher MYC expression than those without by immunohistochemistry staining.

median $\mathrm{H}$ score 22 vs. $13.5, \mathrm{p}<0.001$

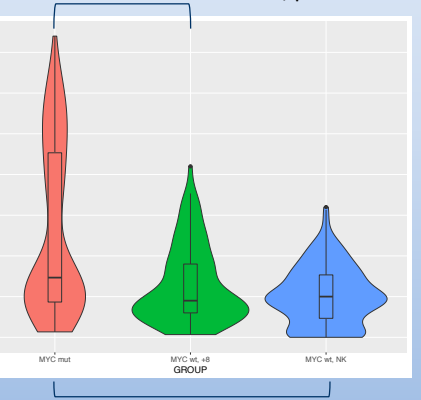

median $\mathrm{H}$ score 22 vs. $15, \mathrm{p}<0.001$ )

\section{Conclusions}

- We identified significant association between mutations and certain clinical phenotype.

Class 1 mutations (NPM1, FLT3, PTPN11, NRAS) were associated with proliferative disease (high WBC, blast, LDH), whereas patients with mutations in TP53, STAG2,

IDH1, IDH2, and NPM1 mutations were associated with decreased expression of HLA-DR. Mutations associated with NOTCH or RAS-RTK pathway showed increased ation hematopoietic differentiation transcription factor showed decreased expression of myeloid markers. MYC mutations were associated with MYC protein overexpression in AML. 